\title{
A novel genetic variant of the goat Six6 gene and its association with production traits in Chinese goat breeds
}

\author{
C.Y. Pan ${ }^{1,2}$, X.Y. Lan ${ }^{1,2}$, H.Y. Zhao', S.R. Hu ${ }^{2}$, Y.T. Huai ${ }^{2}$, C.Z. Lei ${ }^{2}$ and \\ H. Chen ${ }^{2}$ \\ ${ }^{1}$ College of Life Sciences, Northwest A\&F University, Yangling, \\ Shaanxi, China \\ ${ }^{2}$ College of Animal Science and Technology, Northwest A\&F University, \\ Yangling, Shaanxi, China \\ Corresponding authors: X.Y. Lan / H. Chen \\ E-mail: lanxianyong79@yahoo.com.cn / 1lxxyyease@126.com
}

Genet. Mol. Res. 10 (4): 3888-3900 (2011)

Received June 15, 2011

Accepted October 19, 2011

Published November 22, 2011

DOI http://dx.doi.org/10.4238/2011.November.22.9

\begin{abstract}
We looked for novel genetic variations within the Six6 gene by PCR-SSCP, DNA sequencing and forced RFLP-PCR and estimated their associations with production traits in 2132 goats of eight indigenous Chinese breeds. A novel single nucleotide polymorphism (NM_001104993.1: g.232T >C) within the goat Six6 gene was identified. The frequencies of allele " $C$ " varied from 0.8621 to 1.000 , which were in Hardy-Weinberg equilibrium. Genotype and allele frequencies were found to be significantly different in the breeds. Association analysis revealed a significant relationship between genotypes and cannon circumference in Hainan black goats $(P=0.047)$; adult individuals with genotype CT showed higher cannon circumference than those with genotype CC. Another significant association of genotypes with fiveyear-old fiber length was found in Inner Mongolia white cashmere goats $(\mathrm{P}=0.002)$. In addition, individuals with genotype CT had longer fiber length than those with genotype CC. The data revealed that the Six6
\end{abstract}


gene positively affects growth traits and cashmere traits. Polymorphism of Six6-PstI could be useful as a DNA marker for goat breeding and genetics via marker-assisted selection.

Key words: Goat; Sine oculis homeobox homolog 6 (Six 6$)$ gene; Genetic variation; Production traits; Association analysis

\section{INTRODUCTION}

Compared with the traditional animal breeding methods, marker assisted selection (MAS), a process whereby a DNA marker is used for indirect selection of a genetic determinant or determinants of a trait of interest, is more powerful and efficient (Pedersen et al., 2009). Much later, there initially emerged in genetic epidemiology, a genome-wide association study (GWA study, or GWAS), which is an examination of all or most of the genes (the genome) of different individuals of a particular species to see to what extent the genes vary from individual to individual. Different variations are then associated with different traits, such as diseases (Petrie et al., 2011). Some animal breeders performed this method to analyze animal production traits related genes for several animal production traits (Sahana et al., 2011). However, until now, few studies have focused on GWA analysis of production traits in the goat, which may due to the following four reasons. Firstly, DNA sequences of goat genomics have not been accurately published. Secondly, GWA studies search the entire genome for associations rather than focusing on small candidate areas, and this aspect of GWA has attracted the criticism as being expensive "factory science". Thirdly, the GWA approach can also be problematic because the massive number of statistical tests performed presents an unprecedented potential for false-positive results. Fourthly, there are more unresolved problems as viewed from the inferential point of view. Specifically, a number of classification problems have still not been adequately solved (Lasky-Su et al., 2010).

Considering the cost and time, for goat breeding and genetics, MAS is now considered the optimal choice (Weller and Ron, 2011). Unfortunately, little information about the candidate genes affecting production traits is available. For this reason, excavating the suitable candidate DNA markers that correlate with growth and Cashmere traits in meat and Cashmere goat breeds has become a major objective. For this approach, DNA sequencing and PCRRFLP are convenient and helpful.

The Sine oculis homeobox homolog (Six) gene family encodes a group of transcription factors that is related to homeobox-containing gene product in vertebrates by two conserved domains (Conte et al., 2005; Diaczok et al., 2008; Weasner and Kumar, 2009). Previous studies have reported that the Six family plays an important role in the entire development of the fly's visual system (Cheyette et al., 1994; Jean et al., 1999). Sine oculis homeobox homolog 6 (Six6), also known as Optx2, Six9, was expressed in the embryonic pituitary and therefore was implicated in its development (Diaczok et al., 2005; Rachel et al., 2011). Earliest Six6 expression was detected in the floor of the diencephalic portion of the primitive forebrain, a region predicted to give rise to the neurohypophysis and to the hypothalamus. Later on, Six6 mRNA was found in the primordial tissues giving rise to mature pituitary: the Rathke's pouch and the infundibular recess (Conte et al., 2005). In the optic primordium, Six6 demarcates the presumptive ventral optic stalk and the ventral portion of the future neural retina (Jeans et al., 2006). In the develop- 
ing eye, Six 6 expression was detected in the neural retina, the optic chiasma and optic stalk, but not in the lens. Accordingly, the Six 6 gene was expressed in a more restricted fashion, being detected in the developing hypothalamus, pituitary, eyes, and optic stalk of rodents (Jean et al., 1999; Rachel et al., 2011). The reported studies have demonstrated that animals lacking Six6 have retinal and optic nerve defects and hypoplastic pituitaries (Rachel et al., 2011). During normal development in mammals, Six 6 was proposed to repress those genes which encoding inhibitors of the cell cycle, thereby promoting precursor cell proliferation in the developing retina and pituitary (Tétreault et al., 2009; Rachel et al., 2011). In other words, Six6 represents as the earliest genetic markers for pituitary development, suggesting that this gene could be a candidate gene for studying association with production traits in mammals.

To date, little information about Six 6 gene affecting growth traits and cashmere traits is available in livestock except bovine (Huai et al., 2011). In 2011, Huai et al. revealed a novel mutation (NC_007308: g 2015T > C) in the TGA stop codon of a bovine Six6 gene, which leads to an ORF shift and extension of the encoded protein for four amino acids (Arg ${ }^{223}$ $\left.\mathrm{Gln}^{224}-\mathrm{Arg}^{225}-\mathrm{Val}^{226}\right)$. Although no statistically significant associations of bovine Six6 gene with growth traits were detected, a novel mutation in the TGA stop codon also implied that this mutation might affect other biophysical and biochemical indexes. Apart from this study of bovine Six6 gene, there was no information about genetic variation of Six6 gene and their effects on production traits in goat.

Therefore, it is an interesting and meaningful work to scan unreported genetic variations within Six6 gene and evaluate their associations with growth traits and cashmere traits in goat. In this study, PstI polymorphisms within goat Six6 gene was identified, its significant association with growth traits and cashmere traits was analyzed, which provided evidence that Six6 gene can be a genetic marker for goat selection and breeding through MAS.

\section{MATERIAL AND METHODS}

\section{Collection of DNA samples and data of goat product traits}

Blood samples and ear tissues samples were obtained from 2132 healthy and unrelated female individuals from eight Chinese indigenous goat breeds. Guanzhong dairy goat (GZ, dairy breed, $N=658$ ) and Xinong Sannen dairy goat (XNSN, dairy breed, $N=267$ ) were from native breeding farms in Sanyuan County, Qianyang County, Shaanxi Province, respectively. Inner Mongolia white cashmere goat (IMWC, meat and cashmere breed (companion utility), $\mathrm{N}$ $=573$ ), Shaanbei white cashmere goat (SBWC, cashmere breed, $\mathrm{N}=201$ ) and Xinjiang white cashmere goat (XJWC, cashmere breed, $\mathrm{N}=93$ ), were from native breeding farms in Inner Mongolia autonomous region, Shaanxi Province, Xinjiang autonomous region, respectively. Hainan black goat (HNBG, meat breed, $\mathrm{N}=284)$, Xuhuai white goat $(\mathrm{XH}$, meat breed, $\mathrm{N}$ $=35)$ and Banjiao goat (BJ, meat breed, $\mathrm{N}=21$ ) were from Hainan, Jiangsu and Guizhou provinces, respectively. Genomic DNAs were extracted from leucocytes and ear tissues using salt-chloroform extraction protocol (Sambrook and Russell, 2001).

It should be noted that IMWC goats were from three different places under uniform feed and management. They were derived from 10 sires more than one-year old. In fact, most of them were six-years old. Data of body weight, cashmere yields, and fiber length and wool thickness collected from the herd between 2000 and 2007 were used in association analysis. 
Mating of IMWC goats occurred over 60-90 days (normally from September to November), and then parturition occurred the following March to May. Kids were weaned at 4 months of age. Weaning weight was measured to the nearest $0.01 \mathrm{~kg}$. All animals were individually identified at birth. Identification number, date of birth, type of birth, sex, body weight at birth and weaning were recorded. Pedigree information on the sires and dams, and the age of the dams were recorded. Cashmere yield data were harvested once a year by shearing in May, and weighed using an electronic scale following harvest. The fiber length was measured in May of each year as well as wool thickness. A fiber length analyzer was used in the Wool Analysis Laboratory of Inner Mongolia Agricultural University as well as wool thickness. Fiber length and wool thickness were measured to the nearest $1 \mathrm{~mm}$ (Lan et al., 2007, 2009).

Additionally, the HNBG was reared in native breeding farms, in Zanzhou County (East Longitude 109.5 degree; North Latitude 19.5 degree), Hainan Province, China. All goats were adult goats (2-3 years old). Measurements were taken from 284 individuals, involving in body weight $(\mathrm{BW}, \mathrm{kg})$, body height $(\mathrm{BH}, \mathrm{cm})$, body length $(\mathrm{BL}, \mathrm{cm})$, chest circumference $(\mathrm{ChC}, \mathrm{cm})$, chest depth $(\mathrm{ChD}, \mathrm{cm})$, chest width $(\mathrm{ChW}, \mathrm{cm})$, hucklebone width $(\mathrm{HuW}, \mathrm{cm})$ and cannon circumference $(\mathrm{CaC}, \mathrm{cm})$. Measurements were obtained by the standard measurements of meat goat. In addition, body length index (BLI, \%), chest circumference index (ChCI, \%), cannon circumference index (CaCI, \%), Trunk index (TI, \%), Chest width index (CWI, \%) and Hucklebone width index (HuWI, \%) were also calculated.

\section{Polymerase chain reaction (PCR) amplification}

As the goat Six6 gene sequence was not available, three pairs of primer sequences were designed to scan polymorphism from different amplification PCR products including $5^{\prime}$ UTR, exons, introns and 3' UTR within goat Six6 gene based on bovine Six6 gene sequence $\left(\right.$ GenBank $^{\circledR}$ sequence NC_007308, NM_001104493.1, Table 1).

\begin{tabular}{|c|c|c|c|c|c|}
\hline Loci & Primer sequences & $\mathrm{T}_{\mathrm{m}}\left({ }^{\circ} \mathrm{C}\right)$ & Sizes and location & $\begin{array}{l}\text { GenBank }^{\mathbb{B}} \\
\text { Acc. No. }\end{array}$ & $\begin{array}{l}\text { Detection } \\
\text { methods }\end{array}$ \\
\hline P1 & $\begin{array}{l}\text { F:5'-TCCTCCAATCCCTCCCG-3' } \\
\text { (nt 75-91) } \\
\text { R:5'-CGTTCAAGGCTCGGAGGTCTAT-3' } \\
\text { (nt 835-58) }\end{array}$ & 60.0 & $\begin{array}{l}784 \text { bp, including } \\
\text { 5'UTR,E1,E2,IVS1,IVS2 }\end{array}$ & $\begin{array}{l}\text { NC_007308 } \\
\text { (nt75-858) }\end{array}$ & $\begin{array}{l}\text { DNA pool } \\
\text { sequencing }\end{array}$ \\
\hline P2 & $\begin{array}{l}\text { F:5'-TCCTCCAATCCCTCCCG-3' } \\
\text { (nt 75-91) } \\
\text { R-new:5'-AACATCGAGGCAGCGCCGGCGACTGC-3' } \\
\text { (nt 233-258) }\end{array}$ & 51.3 & $\begin{array}{l}184 \text { bp including } \\
\text { 5'UTR, E1,IVS1 }\end{array}$ & $\begin{array}{l}\text { NM_001104993.1 } \\
\text { (nt75-258) }\end{array}$ & $\begin{array}{l}\text { PstI forced } \\
\text { PCR-RFLP }\end{array}$ \\
\hline P3 & $\begin{array}{l}\text { F:5'-GCTGTGGCTCGAAGCTCATTAC-3' } \\
\text { (nt518-79) } \\
\text { R:5'-TGTAGCCTTGGTATGGTGACTG-3' } \\
\text { (nt1046-67) }\end{array}$ & 58.0 & $\begin{array}{l}550 \text { bp including E2, } \\
\text { IVS1, IVS2 }\end{array}$ & $\begin{array}{l}\text { NC_007308 } \\
\text { (nt518-1067) }\end{array}$ & $\begin{array}{l}\text { PCR-SSCP; } \\
\text { DNA sequencing }\end{array}$ \\
\hline P4 & $\begin{array}{l}\text { F:5'-GGGCTGACTGCTGGGCTTA-3' } \\
\text { (nt1764-82) } \\
\text { R:5'-AGACCAAGCAACCCAGCG-3' } \\
\text { (nt2130-47nt) }\end{array}$ & 65.5 & $\begin{array}{l}\text { 384bp including E3, } \\
\text { IVS2, 3'UTR }\end{array}$ & $\begin{array}{l}\text { NC_007308 } \\
\text { (nt1764-2147) }\end{array}$ & $\begin{array}{l}\text { PCR-SSCP; } \\
\text { DNA sequencing }\end{array}$ \\
\hline
\end{tabular}

"E” means exon; "IVS" means intron; "UTR" means untranscriptional region; "Acc. No." means accession number.

The $25-\mu \mathrm{L}$ PCR volume contained $50 \mathrm{ng}$ genomic DNA, $0.5 \mu \mathrm{M}$ of each primer, $1 \mathrm{X}$ buffer (including $1.5 \mathrm{mM} \mathrm{MgCl}$ ), $200 \mu \mathrm{M}$ dNTPs (dATP, dTTP, dCTP and dGTP), and 0.625 
U Taq DNA polymerase (MBI, Vilnius, Lithuania). The cycling protocol was 4 min at $95^{\circ} \mathrm{C}$, 35 cycles of denaturing at $94^{\circ} \mathrm{C}$ for $30 \mathrm{~s}$, annealing for $30 \mathrm{~s}$, extending at $72^{\circ} \mathrm{C}$ for $30 \mathrm{~s}$, with a final extension at $72^{\circ} \mathrm{C}$ for $10 \mathrm{~min}$.

\section{Detecting genetic variation by PCR-SSCP and DNA sequencing}

Based on the fragment size of PCR products, PCR products of P3 and P4 loci within goat Six6 gene were analyzed for single-strand conformation polymorphisms (SSCP). Aliquots of $5 \mu \mathrm{L}$ PCR products from different individuals were mixed with $5 \mu \mathrm{L}$ denaturing solution (95\% formamide, $25 \mathrm{mM}$ EDTA, $0.025 \%$ Xylene-Cyanole and $0.025 \%$ bromophenol blue), heated for $10 \mathrm{~min}$ at $98^{\circ} \mathrm{C}$ and chilled on the ice. Denatured DNA was subjected to PAGE $(80 \times 73 \times 0.75 \mathrm{~mm})$ in $1 \mathrm{X}$ TBE buffer and constant voltage $(200 \mathrm{~V})$ for $2.5 \mathrm{~h}$ (Lan et al., 2007). The gel was stained with $0.1 \%$ silver nitrate. At least three different PCR products which demonstrated the same polymorphic patterns on SSCP gels were randomly selected to purify and sequence with the forward and reverse directions. Nucleotide sequence alignments, translations and comparisons were carried out using the Megalign software module of DNASTAR (version 4.0) and BioXM software (version 2.6).

\section{Genotyping of PstI polymorphism by DNA pool sequencing and Forced RFLP-PCR}

Since the SSCP method is expensive, complicated and time-consuming, DNA pool sequencing had also been explored as a helpful strategy to detect SNPs and small inserts/deletions (indels) for a long PCR fragment, and combined forced RFLP-PCR to characterize the types of DNA polymorphism (Sham et al., 2002; Betty et al., 2004). Forced RFLP-PCR is a method that is achieved by introducing a point mutation into one of primer sequences, so that the PCR product will contain a restriction endonuclease recognition site. Thus, the PCR products from carrier animals contained a restriction site, as well as non carrier individuals, without restriction site.

Different DNA samples of unknown sequences were pooled in same ratio mixtures. For each breed pool, the DNAs were pooled and then amplified and sequenced for the special primer pairs (P1 locus). DNA pool sequencing and Blastn analysis revealed a novel mutation NM_001104993.1:g.232T >C in Six6 gene. As this mutation could not be recognized by common enzymes, a piece of new reverse primer (P2-R: 5'-AACATCGAGGCAGCGCCGGCGACTGC3', nt 233-258, GenBank ${ }^{\circledR}$ accession No. NM_001104993.1) was designed, where the actual nucleotide "T" was induced into " $A$ ". The reverse primer was deliberately introduced by a point mutation which would create a PstI restriction site (CTGCAG) in PCR products from g.232T goat carrier. In contrast, PCR products from the g.232C non carriers lacked this site. This novel mutation of goat Six 6 gene can be genotyped by PstI forced PCR-RFLP. The $20 \mu \mathrm{L}$ PCR products were digested with $10 \mathrm{U}$ Pst (MBI, Vilnius, Lithuania) for $5 \mathrm{~h}$ at $37^{\circ} \mathrm{C}$ following the supplier protocol. The digested products were following detected by electrophoresis in 4.0 $\%$ agarose gel and stained with ethidium bromide.

\section{Statistical analysis}

Genotypic frequencies, allelic frequencies and Hardy-Weinberg Equilibrium were directly calculated. Population genetic indexes, involving in gene heterozygosity and homo- 
zygosity, effective allele numbers and PIC (Polymorphism Information Content), were calculated by Nei's method (Nei and Li, 1979). Differences for these frequencies at goat Six6-PstI locus among/between Chinese breeds were analyzed using the Chi-test, which were performed by SPSS software (version 18.0).

The independent Chi-square t-test procedure of SSPS software (version 18.0) was adopted to examine the experimental data of HNBG adult goats. Body weight and other growth traits association analysis was based on the model: $\mathrm{Y}_{i j}=\mu+$ Genotype $_{i}+\mathrm{E}_{i j}$, where $Y_{i j}$ was the observation of animal, $\mu$ was the overall mean, genotype ${ }_{i}$ was the fixed effect of the genotype, and $E_{i j}$ was the random effect. Effects associated with some of the factors, were not included in the above mentioned models due to preliminary statistical analysis indicating that these factors were not significant sources of variation in female populations. In addition, farm and sex effects were not considered, because all animals were female and kept in the same farm. Significant difference was stated when the genotype effect was a significant source of variation, and the P-value for the difference between the least square means for each genotype was less than $\mathrm{P}=0.05$ level.

SPSS software (version 18.0) was applied to carry out the statistical analysis. Statistical analysis was performed on the basis of records of body weight, status, body length, heart girth, cashmere yields, and fiber length and wool thickness in goats. An effect associated with farm, sex and season of birth (spring versus fall) was not included in the linear model, as the preliminary statistical analyses indicated that these effects did not have a significant influence on variability of traits in the female IMWC population. Consequently, the following model was used to analyze the association of different genotypes with performance traits (Lan et al., 2007): $\mathrm{Y}_{\mathrm{iklm}}=\mu+\mathrm{S}_{\mathrm{i}}+\mathrm{A}_{\mathrm{k}}+\mathrm{G}_{1}+(\mathrm{AG})_{\mathrm{kl}}+\mathrm{E}_{\mathrm{iklm}}$, where $Y_{i k l m}$ was the trait measured on each of the iklm ${ }^{\text {th }}$ animal, $\mu$ was the overall population mean, $S_{i}$ was the fixed effect associated with the $\mathrm{i}^{\text {th }}$ sire, $A_{k}$ was fixed effect due to the $\mathrm{k}^{\text {th }}$ age, $G_{l}$ was the fixed effect associated with $1^{\text {th }}$ genotype, $(A G)_{k l}$ was interaction between the $\mathrm{k}^{\text {th }}$ age and the $1^{\text {th }}$ genotype and $E_{i k l m}$ was the random error.

\section{RESULTS}

\section{A novel genetic variation of the goat Six6 gene}

PCR-SSCP analysis showed monopolymorphism in P3 and P4 locus (Figures no shown). While polymorphic DNA sequences were revealed by DNA pool sequencing and forced RFLP-PCR. A novel genetic variation within goat Six6 gene (NM_001104993.1:g.232T $>$ C locating on $-22 \mathrm{C}>\mathrm{T}$ in the upstream) was revealed (Figure 1). A total of two genotypes in Pst $\mathrm{I}$ locus were identified (Figure 2).

\section{Genotypic and allelic frequencies, genetic indexes analysis}

From Table 2, frequencies of popular allele "C" in eight goat breeds varied from 0.8621 to 1.000 . According to Nei's methods, the population genetic indexes involving in gene homozygosity, gene heterozygosity, effective allele numbers and polymorphism information content (PIC) are also listed in Table 3. All analyzed breeds were at the Hardy-Weinberg equilibrium $(\mathrm{P}>0.05)$. 


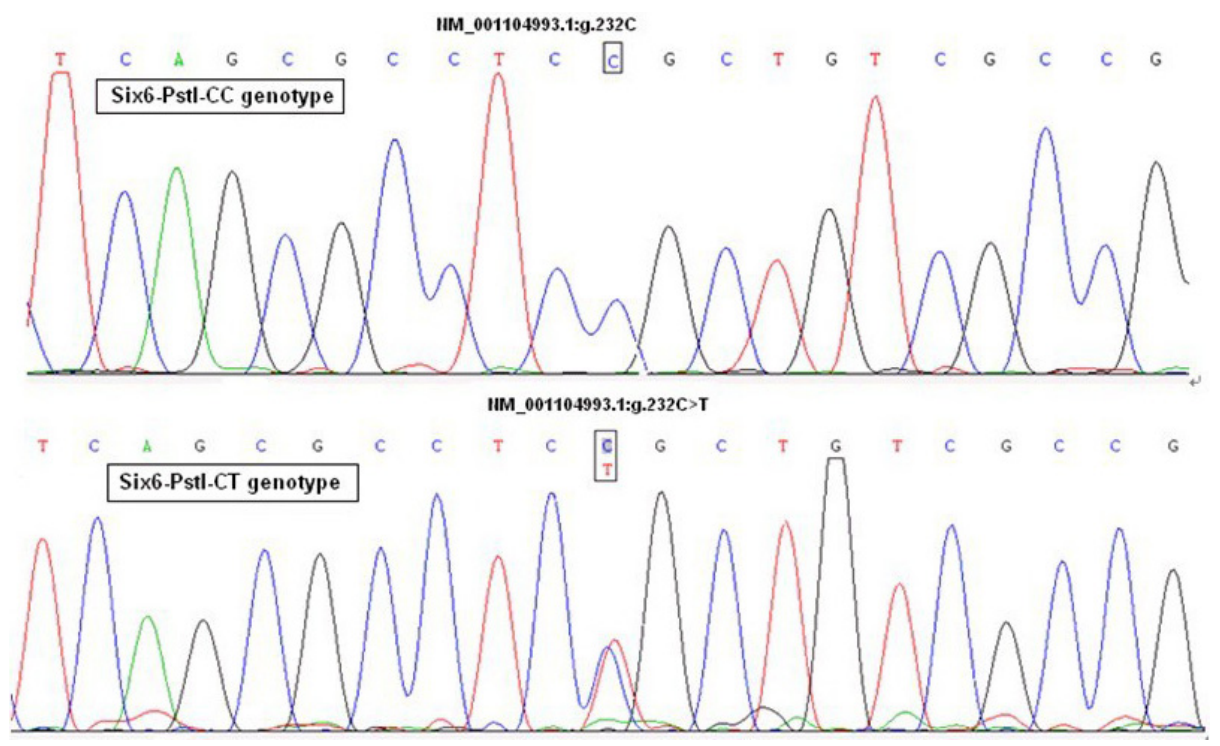

Figure 1. Sequence maps of PstI locus within goat Six6 gene.

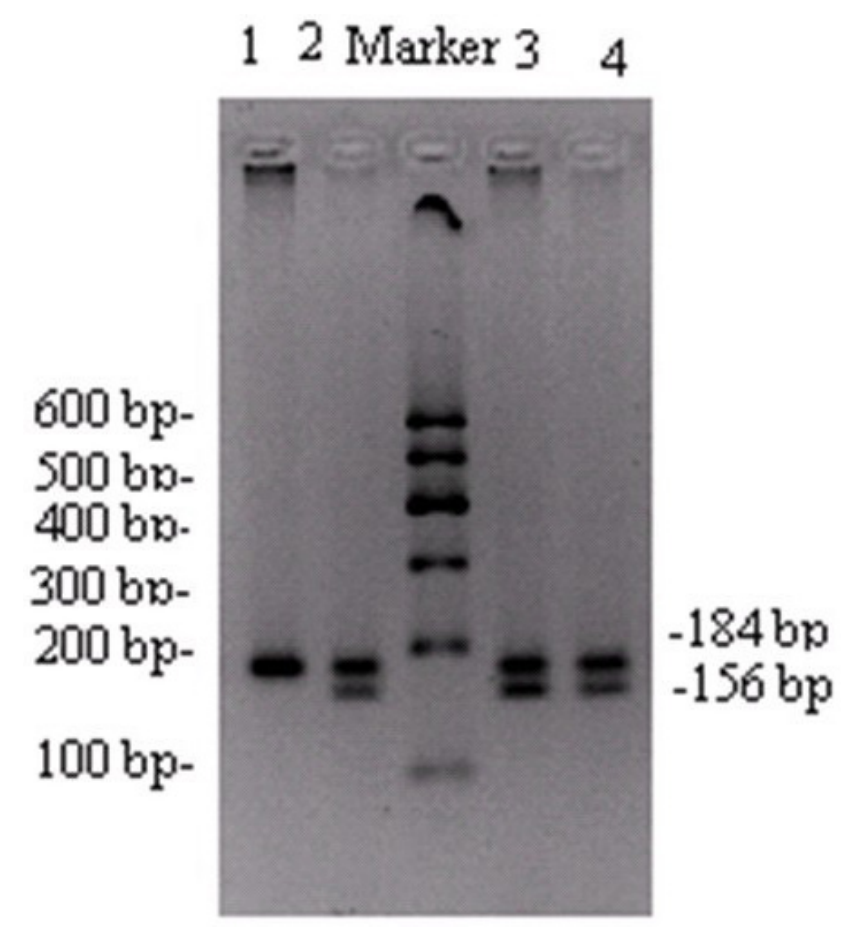

Figure 2. Electrophoresis patterns of Pst forced PCR-RFLP analysis within goat Six 6 gene. Lane $1=\mathrm{CC}$ genotype; Lane $2-4=\mathrm{CT}$ genotype; CC genotype $=184 \mathrm{bp} ; \mathrm{TC}$ genotype $=184 \mathrm{bp}, 156 \mathrm{bp}$ and $28 \mathrm{bp} ; \mathrm{CC}$ genotype $=156 \mathrm{bp}$ and $28 \mathrm{bp}$. 
Table 2. Genotypic and allelic frequencies of PstI polymorphism within goat Six6 gene in goats.

\begin{tabular}{|c|c|c|c|c|c|c|c|}
\hline \multirow[t]{2}{*}{ Breeds } & \multirow[t]{2}{*}{ Total } & \multicolumn{3}{|c|}{ Observed genotypes and frequencies } & \multicolumn{2}{|c|}{ Allelic frequencies } & \multirow[t]{2}{*}{ Utility types } \\
\hline & & $\mathrm{CC}\left(\mathrm{P}_{\mathrm{CC}}\right)$ & $\mathrm{CT}\left(\mathrm{P}_{\mathrm{CT}}\right)$ & $\mathrm{TT}\left(\mathrm{P}_{\mathrm{TT}}\right)$ & $\mathrm{C}\left(\mathrm{P}_{\mathrm{C}}\right)$ & $\mathrm{T}\left(\mathrm{P}_{\mathrm{T}}\right)$ & \\
\hline GZ & 658 & $632(0.9608)$ & $26(0.0392)$ & $0(0)$ & 0.9804 & 0.0196 & Dairy utility \\
\hline XNSN & 267 & $267(1.000)$ & $0(0)$ & $0(0)$ & 1.0000 & 0 & Dairy utility \\
\hline IMWC & 573 & $416(0.7260)$ & $156(0.2723)$ & $1(0017)$ & 0.8621 & 0.1379 & Cashmere and meat (companion) \\
\hline SBWC & 201 & $183(0.9104)$ & $18(0.0896)$ & $0(0)$ & 0.9552 & 0.0448 & Cashmere utility \\
\hline XJWC & 93 & $73(0.7849)$ & $20(0.2151)$ & $0(0)$ & 0.8925 & 0.1075 & Cashmere utility \\
\hline HNBG & 284 & $260(0.9167)$ & $24(0.0833)$ & $0(0)$ & 0.9583 & 0.0417 & Meat utility \\
\hline $\mathrm{XH}$ & 35 & $32(0.9143)$ & $3(0.0857)$ & $0(0)$ & 0.9571 & 0.0429 & Meat utility \\
\hline BJ & 21 & $19(0.9048)$ & $2(0.0952)$ & $0(0)$ & 0.9524 & 0.0476 & Meat utility \\
\hline Total & 2132 & $1883(0.8830)$ & $248(0.1165)$ & $1(0.0005)$ & 0.9413 & 0.0587 & \\
\hline
\end{tabular}

$\mathrm{GZ}=$ Guanzhong dairy goat; XNSN = Xinong Sannen dairy goat; IMWC = Inner Mongolia white cashmere goat; SBWC = Shaanbei white cashmere goat $(\mathrm{SBWC}) ; \mathrm{XJWC}=$ Xinjiang white cashmere goat; $\mathrm{HNBG}=$ Hainan blacke goat; $\mathrm{XH}=$ Xuhuai white goat; $\mathrm{BJ}=$ Banjiao goat.

Table 3. Genetic indexes of PstI polymorphism within goat Six6 gene in goat.

\begin{tabular}{|c|c|c|c|c|c|c|}
\hline Breeds & $\mathrm{H}_{\mathrm{e}}$ & $\mathrm{H}_{\mathrm{o}}$ & $\mathrm{N}_{\mathrm{e}}$ & PIC & Allele variations & HWE \\
\hline GZ & 0.9613 & 0.0387 & 1.0403 & 0.0380 & $1.5 \times 10^{-5}$ & $\mathrm{P}>0.05$ \\
\hline XNSN & 1.0000 & 0 & 1.0000 & 0 & 0 & $\mathrm{P}>0.05$ \\
\hline IMWC & 0.7623 & 0.2377 & 1.3119 & 0.2095 & 0.00010 & $\mathrm{P}>0.05$ \\
\hline SBWC & 0.9145 & 0.0855 & 1.0935 & 0.0819 & 0.00011 & $\mathrm{P}>0.05$ \\
\hline XJWC & 0.8081 & 0.1919 & 1.2375 & 0.1735 & 0.00052 & $\mathrm{P}>0.05$ \\
\hline HNBG & 0.9191 & 0.0809 & 1.0881 & 0.0777 & $7.1 \times 10^{-5}$ & $\mathrm{P}>0.05$ \\
\hline $\mathrm{XH}$ & 0.9180 & 0.0820 & 1.0894 & 0.0787 & 0.00060 & $\mathrm{P}>0.05$ \\
\hline BJ & 0.9093 & 0.0907 & 1.0998 & 0.0866 & 0.00113 & $\mathrm{P}>0.05$ \\
\hline Total & 0.8896 & 0.1104 & 1.1241 & 0.1043 & $1.3 \times 10^{-5}$ & $\mathrm{P}>0.05$ \\
\hline
\end{tabular}

$\mathrm{H}_{\mathrm{e}}=$ gene heterozygosity; $\mathrm{H}_{\mathrm{o}}=$ gene homozygosity; $\mathrm{N}_{\mathrm{e}}=$ effective allele numbers; $\mathrm{PIC}=$ polymorphism information content; HWE $=$ Hardy-Weinberg equilibrium.

\section{Relationship between genetic variation and production traits}

The establishment of relationships between different genotypes and body weight, body sizes was performed in HNBS goat (Table 4). Association analysis revealed a significant statistical relationship between different genotypes and cannon circumference $(\mathrm{CaC})$ in HNBS goats $(\mathrm{P}=0.047)$. The adult individuals with genotype $\mathrm{CT}$ showed higher $\mathrm{CaC}$ than those with genotype CC. No significant relationships between polymorphism of PstI locus with body weight and other growth traits were detected. However, there were no significant relationships between different genotypes of Pst locus within Six6 gene and body weights of 1-7 years old individuals in IMWC goats (data not shown).

From Table 5, the establishment of relationships between different genotypes and cashmere traits was performed in IMWC. There were no significant relationships between polymorphisms and cashmere traits except five year old fiber length. In detailed, a significant statistical association of different genotypes with 5 year-old fiber length was revealed $(\mathrm{P}=0.002)$. The individuals with genotype $\mathrm{CT}$ demonstrated longer fiber length than those with genotype $\mathrm{CC}$ in five year old IMWC goats. 
Table 4. Relationship between PstI polymorphism within Six6 gene and growth tratis in Hainan black goat.

\begin{tabular}{|c|c|c|c|}
\hline \multirow[t]{2}{*}{ Growth traits } & \multicolumn{2}{|c|}{ Observed genotypes (Mean \pm SD) } & \multirow[t]{2}{*}{$\mathrm{P}$ values (two-tailed) } \\
\hline & $\mathrm{CC}(\mathrm{N}=260)$ & $\mathrm{CT}(\mathrm{N}=24)$ & \\
\hline $\mathrm{BW}(\mathrm{kg})$ & $28.24 \pm 0.44$ & $28.67 \pm 1.63$ & $\mathrm{P}=0.775$ \\
\hline $\mathrm{BH}(\mathrm{cm})$ & $52.55 \pm 0.26$ & $53.54 \pm 1.09$ & $\mathrm{P}=0.384$ \\
\hline $\mathrm{BL}(\mathrm{cm})$ & $55.86 \pm 0.29$ & $55.85 \pm 0.93$ & $\mathrm{P}=0.995$ \\
\hline $\mathrm{ChC}(\mathrm{cm})$ & $71.90 \pm 0.42$ & $72.79 \pm 1.27$ & $\mathrm{P}=0.520$ \\
\hline $\mathrm{ChD}(\mathrm{cm})$ & $26.37 \pm 0.15$ & $26.87 \pm 0.47$ & $\mathrm{P}=0.334$ \\
\hline $\mathrm{ChW}(\mathrm{cm})$ & $14.81 \pm 0.12$ & $14.85 \pm 0.38$ & $\mathrm{P}=0.910$ \\
\hline $\mathrm{HuW}(\mathrm{cm})$ & $13.64 \pm 0.09$ & $13.77 \pm 0.26$ & $P=0.653$ \\
\hline $\mathrm{CaC}(\mathrm{cm})$ & $7.75 \pm 0.05^{b}$ & $8.06 \pm 0.17^{\mathrm{a}}$ & $* \mathrm{P}=0.047$ \\
\hline BLI (\%) & $128.92 \pm 0.59$ & $130.45 \pm 1.33$ & $P=0.431$ \\
\hline $\mathrm{ChCI}(\%)$ & $106.57 \pm 0.51$ & $104.82 \pm 1.66$ & $\mathrm{P}=0.310$ \\
\hline $\mathrm{CaCl}(\%)$ & $137.03 \pm 0.65$ & $136.53 \pm 2.12$ & $\mathrm{P}=0.820$ \\
\hline TI $(\%)$ & $14.73 \pm 0.10$ & $15.14 \pm 0.30$ & $\mathrm{P}=0.231$ \\
\hline CWI (\%) & $56.28 \pm 0.40$ & $55.30 \pm 1.04$ & $\mathrm{P}=0.454$ \\
\hline HuWI (\%) & $108.9 \pm 0.75$ & $108.16 \pm 2.40$ & $P=0.762$ \\
\hline
\end{tabular}

$\mathrm{BW}=$ Body weight; $\mathrm{BH}=$ Body height $\mathrm{BL}=$ Body length; $\mathrm{ChC}=$ Chest circumference; $\mathrm{ChD}=\mathrm{Chest}$ depth; $\mathrm{ChW}=$ Chest width; $\mathrm{HuW}=$ Hucklebone width $\mathrm{CaC}=$ Cannon circumference; $\mathrm{BLI}=$ Body length index $(\%)$; $\mathrm{ChCI}=$ Chest circumference index $(\%) ; \mathrm{CaCI}=$ Cannon circumference index $(\%)$; $\mathrm{TI}=$ Trunk index $(\%) ; \mathrm{CWI}=$ Chest width index $(\%)$; HuWI $=$ Hucklebone width index $(\%)$; Means superscription with different letters means significant statistical difference $(* \mathrm{P}<0.05$ or $* * \mathrm{P}<0.01) ; \mathrm{SD}=$ standard deviation.

Table 5. Relationship between PstI polymorphism within goat Six6 gene and cashmere traits in the Inner Mongolia white cashmere goat.

\begin{tabular}{|c|c|c|c|c|}
\hline \multirow[t]{2}{*}{ Traits } & \multirow[t]{2}{*}{ Performance traits } & \multicolumn{2}{|c|}{ Observed genotypes $($ Mean $\pm \mathrm{SD})$} & \multirow[t]{2}{*}{$P$ value (two-tailed) } \\
\hline & & $\mathrm{CC}(\mathrm{n})$ & CT (n) & \\
\hline \multirow{7}{*}{ Wool thickness $(\mathrm{cm})$} & $1 \mathrm{yr}$ wool thickness & $5.83 \pm 0.83(\mathrm{~N}=72)$ & $5.82 \pm 0.76(\mathrm{~N}=28)$ & $P=0.947$ \\
\hline & 2 yr wool thickness & $6.02 \pm 0.85(\mathrm{~N}=158)$ & $6.16 \pm 0.88(\mathrm{~N}=48)$ & $P=0.320$ \\
\hline & 3 yr wool thickness & $5.91 \pm 0.81(\mathrm{~N}=165)$ & $6.09 \pm 0.81(\mathrm{~N}=50)$ & $P=0.167$ \\
\hline & 4 yr wool thickness & $5.88 \pm 0.80(\mathrm{~N}=158)$ & $5.85 \pm 0.72(\mathrm{~N}=51)$ & $\mathrm{P}=0.812$ \\
\hline & 5 yr wool thickness & $5.77 \pm 0.80(\mathrm{~N}=85)$ & $5.85 \pm 0.77(\mathrm{~N}=30)$ & $P=0.638$ \\
\hline & 6 yr wool thickness & $5.89 \pm 0.80(\mathrm{~N}=26)$ & $5.64 \pm 0.99(\mathrm{~N}=7)$ & $P=0.505$ \\
\hline & 7 yr wool thickness & $6.21 \pm 1.01(\mathrm{~N}=14)$ & $5.90 \pm 0.42(\mathrm{~N}=5)$ & $P=0.516$ \\
\hline \multirow[t]{7}{*}{ Fiber length $(\mathrm{cm})$} & 1 yr fiber length & $15.81 \pm 3.97(\mathrm{~N}=72)$ & $15.57 \pm 4.26(\mathrm{~N}=28)$ & $P=0.796$ \\
\hline & 2 yr fiber length & $17.97 \pm 3.88(\mathrm{~N}=158)$ & $16.98 \pm 3.88(\mathrm{~N}=48)$ & $\mathrm{P}=0.121$ \\
\hline & 3 yr fiber length & $18.03 \pm 4.22(\mathrm{~N}=165)$ & $19.24 \pm 3.63(\mathrm{~N}=50)$ & $P=0.069$ \\
\hline & 4 yr fiber length & $17.62 \pm 3.39(\mathrm{~N}=158)$ & $17.75 \pm 4.02(\mathrm{~N}=51)$ & $\mathrm{P}=0.828$ \\
\hline & 5 yr fiber length & $16.59 \pm 3.70(\mathrm{~N}=85)^{\mathrm{c}}$ & $19.10 \pm 4.07(\mathrm{~N}=30)^{\mathrm{a}}$ & $* * \mathrm{P}=0.002$ \\
\hline & 6 yr fiber length & $16.88 \pm 3.24(\mathrm{~N}=26)$ & $14.29 \pm 4.61(\mathrm{~N}=7)$ & $\mathrm{P}=0.095$ \\
\hline & 7 yr fiber length & $17.50 \pm 3.16(\mathrm{~N}=14)$ & $17.60 \pm 3.36(\mathrm{~N}=5)$ & $\mathrm{P}=0.953$ \\
\hline \multirow[t]{7}{*}{ Cashmere yield (g) } & $1 \mathrm{yr}$ cashmere yield & $632.1 \pm 119.5(\mathrm{~N}=72)$ & $638.7 \pm 110.2(\mathrm{~N}=28)$ & $P=0.741$ \\
\hline & 2 yr cashmere yield & $638.7 \pm 126.1(\mathrm{~N}=158)$ & $635.5 \pm 118.2(\mathrm{~N}=48)$ & $\mathrm{P}=0.875$ \\
\hline & 3 yr cashmere yield & $592.9 \pm 135.8(\mathrm{~N}=165)$ & $582.3 \pm 119.0(\mathrm{~N}=50)$ & $P=0.618$ \\
\hline & 4 yr cashmere yield & $594.0 \pm 101.4(\mathrm{~N}=158)$ & $575.8 \pm 106.8(\mathrm{~N}=51)$ & $P=0.274$ \\
\hline & 5 yr cashmere yield & $601.3 \pm 118.7(\mathrm{~N}=85)$ & $605.8 \pm 148.7(\mathrm{~N}=30)$ & $P=0.869$ \\
\hline & $6 \mathrm{yr}$ cashmere yield & $560.2 \pm 111.7(\mathrm{~N}=26)$ & $567.3 \pm 90.2(\mathrm{~N}=7)$ & $\mathrm{P}=0.878$ \\
\hline & 7 yr cashmere yield & $712.4 \pm 159.8(\mathrm{~N}=14)$ & $700.4 \pm 155.9(\mathrm{~N}=5)$ & $\mathrm{P}=0.886$ \\
\hline
\end{tabular}

Means superscription with different letters means significant statistical difference $(* \mathrm{P}<0.05$ or $* * \mathrm{P}<0.01) ; \mathrm{SD}=$ standard deviation.

\section{DISCUSSION}

During mammalian pituitary development, numerous transcription factors involving in Six6, Hesx1, Lhx3, Lhx4, Prop1, Pou1f1, and functional hormones GH, PRL, TSH- $\beta$ play 
several critical roles and are characterized by having their own temporal and spatial expression (Pfäffle and Klammt, 2011). As a member of the POU-domain family, Poulf1 is responsible for a phenotype of combined pituitary hormone deficiency (CPHD) involving GH, PRL and TSH- $\beta$ (Li et al., 1990). A product of the Poulf1 gene regulates the expression of GH, PRL, and TSH- $\beta$ genes which play important roles in growth, development, laction and metabolism in mammals (Li et al., 1990). Mutations within GH (Gupta et al., 2007; Katoh et al., 2008), PRL (Liang et al., 2006; Wang et al., 2009; Bhattacharya et al., 2011) and TSH genes (Medeiros-Neto et al., 1997) are significantly associated with growth, development, lactation and milk performance traits in animals. Based on the regulation net of Pou1f1-Prop1, Six6 gene regulates the expression of Hesx 1 which controlling the mRNA level of Lhx3 and Lhx4 genes (Hu et al., 2008; Tétreault et al., 2009; Kumar, 2009), while these genes directly or indirectly affect their downstream genes, such as Prop1, Pou1f1, GH, PRL and TSH- $\beta$. All of these data implied that Six6 gene is a candidate gene for considering the relationship between its genetic variations and production traits in MAS. Therefore, this work focuses on identification of possible genetic variation of goat Six6 gene and evaluation of their genetic effects on production traits. PCR-SSCP, DNA pool sequencing and forced PCR-RLPF methods were carried out in this study to detect the polymorphism of goat Six 6 gene.

This study preliminarily provided anovelgenetic variant(NM_001104993.1:g.232C $>$ T) locating on $-22 \mathrm{C}>\mathrm{T}$ in the upstream of Six6 gene. The frequencies of minor allele $\mathrm{T}$ varied from 0 to 0.1379 in three different utilities (dairy, cashmere and meat). This result implied that the goat Six 6 gene is characterized by the low mutation frequency, which complied with high conservation and vital function of vertebrate Six6. Previous studies have indicated that Six6 plays a vital role in retinal and optic nerve defects and hypoplastic pituitaries, and is associated with CPHD and septo-optic dysplasia (SOD). Due to rare incidence of human CPHD and SOD, the mutations of human Six6 gene were very rare and its allelic frequency is also low (Diaczok et al., 2008). Consequently, the putative goat CPHD and its related genes, such as bovine Six6, goat Hesxl, goat Propl, goat $L h x 4$, reasonably demonstrated low frequency of minor allele (Sobrier et al., 2006; Li et al., 2008; Lan et al., 2009, 2010; Lai et al., 2009; Huai et al., 2011). According to Nei's methods, the heterozygosity values of different breeds varied from zero to 0.2377 , and their effective allele numbers from 1.000 to 1.3119 . Their PIC values waved from 0 to 0.2095 and average PIC of all analyzed breeds was 0.1043 . In all the breeds, goat Six6 locus possessed low genetic diversity (PIC $<0.250$ ), which was similar to the goat Hesx1. Since the PIC value is an effective variability to assess the genetic diversity from different loci of candidate genes, poor genetic diversity was considered to be characterized in the goat Six6 gene.

In PstI forced-RFLP analysis, relative low CT genotype and no TT genotype were founded in all breeds, which statistically resulted in Hardy-Weinberg equilibrium. In detail, low frequency of allele T below 0.02 is found in GZ (dairy utility) and XNSN (dairy utility) breeds. Relative low frequency of allele T between 0.02 and 0.05 were found in SBWC (cashmere utility), HNBG (meat utility), XH (utility) and BJ (meat utility). However, relative high frequencies of allele T over 0.10 were found in IMWC (cashmere and meat utility) and XJWC (cashmere utility). Accordingly, it was presumed that genotype CT and allele T might be associated with growth and cashmere performance. Further observations by a $\chi^{2}$ test show that genotypic frequencies were found to be significantly different in eight breeds $\left(\chi^{2}=147.674\right.$, df $\left.=6,{ }^{* * *} \mathrm{P}<0.001\right)$, and allelic frequencies in analyzed populations were also found to be sig- 
nificantly different $\left(\chi^{2}=157.250, \mathrm{df}=3,{ }^{* * *} \mathrm{P}<0.001\right)$. Additionally, there were significant differences of genotypic and allelic frequencies distribution between cashmere breed and dairy, meat utilities ( ${ }^{* *} \mathrm{P}<0.01$ or $\left.{ }^{*} \mathrm{P}<0.05\right)$, respectively. Taken together, the observations suggested that the distribution of genotypic and allelic frequencies of goat Six 6 gene was significantly associated with different goat utility (cashmere and meat, dairy breeds). It is possible that different selection diverge affect the frequency of Six6 gene which played multiple roles in meat and cashmere traits.

Association analysis has revealed significant effects of capine Six6 gene on cannon circumference $(\mathrm{CaC})$ and fiber length in meat and cashmere goats. The possible explanations are as follows. Firstly, it might be linked to another mutation in the coding or regulatory regions of the genes which were causal mutations for the growth traits and cashmere traits. GH and PRL genes play multiple important roles in production traits and their SNPs were reported to be frequently in disequilibrium and associatedwith production traits. Probably the novel SNP of Six6 gene performed the same genetic effects of PRL, GH, Pou1f1, and Prop1 genes. Secondly, 5'UTR have been shown to affect transcriptional efficiency of numerous genes in a variety of organisms (Greenwood and Kelsoe, 2003; Sobrier et al., 2006; Kim et al., 2007; Eller et al., 2008; Lawson and Zhang, 2008; Wang et al., 2011).

The genotype $\mathrm{CT}$ showed significantly higher $\mathrm{CaC}$ than $\mathrm{CC}$ in adult goat $(\mathrm{P}<0.05)$, suggesting that allele " $\mathrm{T}$ " produced positive effect on cannon circumference in adult. Consequently, genotype CT was suggested to be utilized to gain higher growth in MAS.

Compared with genotype $\mathrm{CC}$, the genotype CT demonstrated longer fiber length in IMWC goat $(\mathrm{P}<0.05)$, implying that allele " $\mathrm{T}$ " had positive influence on cashmere traits. Genotype CC was suggested to eliminate low wool-length goat in MAS.

\section{CONCLUSION}

In brief, a novel genetic variation (NM_001104993.1:g.232T $>$ C) located on the $5^{\prime}$ UTR of Six6 gene was revealed, which could be genotyped by PstI forced RFLP-PCR, and extended the spectrum of genetic variation. Additionally, significantly associations of this novel genetic variation with growth traits and the Cashmere goat were detected, which is of benefit to goat breeding and genetics through MAS.

\section{ACKNOWLEDGMENTS}

Research supported by the National Natural Science Foundation of China (\#31172184), the Young New Star Project on Science \&Technology of Shaanxi Province (\#2011KJXX, X.Y. Lan), the Natural Science Foundation of Shaanxi Province of China (\#2011JQ3009), the Young Topnotch Researcher Support Project of Northwest A\&F University (\#QNGG-2009007) and Special Fund for animal breeding of the Northwest A\&F University (X.Y. Lan).We thank Professor Jun Luo for collecting dairy goat blood samples and Ph.D graduate T.S. Xu for collecting HNBG goat ear tissues.

\section{REFERENCES}

Betty P, Nathalie I and Jean B (2004). Efficient screening for expressed sequence tag polymorphisms (ESTPs) by DNA pool sequencing and denaturing gradient gel electrophoresis (DGGE) in spruces. Mol. Breed. 13: 263-279. 
Bhattacharya TK, Chatterjee RN, Sharma RP, Niranjan M, et al. (2011). Associations between novel polymorphisms at the 5'-UTR region of the prolactin gene and egg production and quality in chickens. Theriogenology 75: 655-661.

Cheyette BN, Green PJ, Martin K, Garren H, et al. (1994). The Drosophila sine oculis locus encodes a homeodomaincontaining protein required for the development of the entire visual system. Neuron 12: 977-996.

Conte I, Morcillo J and Bovolenta P (2005). Comparative analysis of Six 3 and Six 6 distribution in the developing and adult mouse brain. Dev. Dyn. 234: 718-725.

Diaczok D, Romero C, Zunich J, Marshall I, et al. (2008). A novel dominant negative mutation of OTX2 associated with combined pituitary hormone deficiency. J. Clin. Endocr. Metab. 93: 4351-4359.

Eller A, Branch DW, Nelson L and Silver R (2008). 247: The -634GC polymorphism in the regulatory 5 ' untranslated region (5' UTR) of the vascular endothelial growth factor (VEGF) gene is associated with unexplained recurrent pregnancy loss (RPL). Am. J. Obstet. Gynecol. 199 (Suppl 1): S81.

Greenwood TA and Kelsoe JR (2003). Promoter and intronic variants affect the transcriptional regulation of the human dopamine transporter gene. Genomics 82: 511-520.

Gupta N, Ahlawat SPS, Kumar D, Gupta SC, et al. (2007). Single nucleotide polymorphism in growth hormone gene exon-4 and exon-5 using PCR-SSCP in Black Bengal goats - a prolific meat breed of India. Meat Sci. 76: 658-665.

$\mathrm{Hu}$ S, Mamedova A and Hegde RS (2008). DNA-binding and regulation mechanisms of the SIX family of retinal determination proteins. Biochemistry 47: 3586-3594.

Huai YT, Lan XY, Ma L, Wang J, et al. (2011). Novel mutation in TGA stop-codon of bovine SIX6 gene. Mol. Biol. 45: 218-224.

Jean D, Bernier G and Gruss P (1999). Six6 (Optx2) is a novel murine Six3-related homeobox gene that demarcates the presumptive pituitary/hypothalamic axis and the ventral optic stalk. Mech. Dev. 84: 31-40.

Kim DJ, Park BL, Yoon S, Lee HK, et al. (2007). 5' UTR polymorphism of dopamine receptor D1 (DRD1) associated with severity and temperament of alcoholism. Biochem. Biophys. Res. Commun. 357: 1135-1141.

Kumar JP (2009). The sine oculis homeobox (SIX) family of transcription factors as regulators of development and disease. Cell Mol. Life Sci. 66: 565-583.

Lai X, Lan X, Chen H, Wang X, et al. (2009). A novel SNP of the Hesx 1 gene in bovine and its associations with average daily gain. Mol. Biol. Rep. 36: 1677-1681.

Lan XY, Pan CY, Chen H, Zhang CL, et al. (2007). An AluI PCR-RFLP detecting a silent allele at the goat POU1F1 locus and its association with production traits. Small Rumin. Res. 73: 12.

Lan XY, Pan CY, Li JY, Guo YW, et al. (2009a). Twelve novel SNPs of the goat POU1F1 gene and their associations with cashmere traits. Small Rumin. Res. 85: 116-121.

Lan X, Pan C, Zhang L, Zhao M, et al. (2009b). A novel missense (A79V) mutation of goat PROP1 gene and its association with production traits. Mol. Biol. Rep. 36: 2069-2073.

Lan XY, Lai X, Li ZJ, Wang J, et al. (2010) Effects of genetic variability of the caprine homeobox transcription factor HESX1 gene on performance traits. Mol. Biol. Rep. 37: 441-449.

Lasky-Su J and Lange C (2010). Statistical challenges for genome-wide association studies of suicidality using family data. Eur. Psychiatry 25: 307-309.

Lawson MJ and Zhang L (2008). Housekeeping and tissue-specific genes differ in simple sequence repeats in the 5'-UTR region. Gene 407: 54-62.

Li MJ, Lan XY, Chen H, Zhang LZ, et al. (2008). The novel missense mutation of goat LHX4 gene. Small Rumin. Res. 79: 109-112.

Li S, Crenshaw EB, Rawson EJ, Simmons DM, et al. (1990). Dwarf locus mutants lacking three pituitary cell types result from mutations in the POU-domain gene Pit-1. Nature 347: 528-533.

Liang Y, Cui J, Yang G, Leung FC, et al. (2006). Polymorphisms of 5' flanking region of chicken prolactin gene. Domest. Anim. Endocrinol. 30: 1-16.

Medeiros-Neto G, de Lacerda L and Wondisford FE (1997). Familial Congenital Hypothyroidism Caused by Abnormal and Bioinactive TSH due to Mutations in the beta-Subunit Gene. Trends Endocrinol. Metab. 8: 15-20.

Nei M and Li WH (1979). Mathematical model for studying genetic variation in terms of restriction endonucleases. Proc. Natl. Acad. Sci. U. S. A. 76: 5269-5273.

Pedersen LD, Sorensen AC and Berg P (2009). Marker-assisted selection can reduce true as well as pedigree-estimated inbreeding. J. Dairy Sci. 92: 2214-2223.

Petrie JR, Pearson ER and Sutherland C (2011). Implications of genome wide association studies for the understanding of type 2 diabetes pathophysiology. Biochem. Pharmacol. 81: 471-477.

Pfäffle R and Klammt J (2011). Pituitary transcription factors in the aetiology of combined pituitary hormone deficiency. Best. Pract. Res. Clin. Endocrinol. Metab. 25: 43-60.

Rachel L, Daniel DC, Nichol LGM and Mellon PL (2011). Hypothalamic dysregulation and infertility in mice lacking the 
homeodomain protein Six6. J. Neurosci. 31: 426-438.

Sahana G, Guldbrandtsen B and Lund MS (2011). Genome-wide association study for calving traits in Danish and Swedish Holstein cattle. J. Dairy Sci. 94: 479-486.

Sambrook J and Russell DW (2001). Molecular Cloning: A Laboratory Manual. 3rd edn. Cold Spring Harbor Laboratory Press, New York.

Sham P, Bader JS, Craig I, O’Donovan M, et al. (2002). DNA Pooling: a tool for large-scale association studies. Nat. Rev. Genet. 3: 862-871.

Sobrier ML, Maghnie M, Vie-Luton MP, Secco A, et al. (2006). Novel HESX1 mutations associated with a life-threatening neonatal phenotype, pituitary aplasia, but normally located posterior pituitary and no optic nerve abnormalities. Clin. Endocrinol. Metab. 91: 4528-4536.

Tétreault N, Champagne MP and Bernier G (2009). The LIM homeobox transcription factor Lhx2 is required to specify the retina field and synergistically cooperates with Pax6 for Six6 trans-activation. Dev. Biol. 327: 541-550.

Thomas MG, Enns RM, Shirley KL, Garcia MD, et al. (2007). Associations of DNA polymorphisms in growth hormone and its transcriptional regulators with growth and carcass traits in two populations of Brangus bulls. Genet. Mol. Res. 6: 222-237.

Wang DY, Zhang YJ, Liu YQ and Yang YZ (2009). Karyotype and single nucleotide polymorphism of the prolactin gene in milking bucks. Small Rumin. Res. 87: 96-101.

Wang GB, Li CR, Yang J, Wen PQ, et al. (2011). A regulatory polymorphism in promoter region of TNFR1 gene is associated with Kawasaki disease in Chinese individuals. Hum. Immunol. 72: 451-457.

Weasner BP and Kumar JP (2009). The non-conserved C-terminal segments of Sine Oculis Homeobox (SIX) proteins confer functional specificity. Genesis 47: 514-523.

Weller JI and Ron M (2011). Invited review: quantitative trait nucleotide determination in the era of genomic selection. J. Dairy Sci. 94: 1082-1090. 